\title{
P134 A New Method for Non-invasive Measurement of Arterial Wave Intensity, Speed and Reflection
}

\author{
Ethan Rowland*, Kai Riemer, Kevin Lichtenstein, Mengxing Tang, Peter Weinberg
}

Department of Bioengineering, Imperial College London, UK

\section{ABSTRACT}

Introduction: The ventricles accelerate and decelerate blood; the resulting disturbances propagate through the arterial system as waves. These waves contain clinically useful information: e.g. their magnitude and timing varies with cardiac performance and their speed depends on arterial stiffness. These properties can be studied using Wave Intensity Analysis (WIA) [1] and have been shown to be altered in heart failure [2]. Conventional WIA relies on invasive catheter measurements of blood pressure and velocity. We have developed and validated a new non-invasive ultrasound-based method that allows accurate WIA.

Methods: Employing a novel WIA formulation [3] based on diameter and velocity, and a ultrafast ultrasound imaging system (Verasonics, Kirkland, USA), wave intensity was measured in the abdominal aorta of rabbits. B-mode images were acquired at $1000 \mathrm{~Hz}$, and diameter and velocity measured using standard cross-correlation techniques (the latter after spatio-temporal filtering to enhance the blood signal). Comparative measurements were made with a conventional WIA catheter-based system (Phillips Volcano, San Diego, USA). Ventricular dysfunction was induced by administering esmolol.

Results: Measured non-invasive peak wave intensities showed good agreement with catheter-based ones $(\rho=0.73, p=0.04$ $n=8)$. Changes in the intensity and timing of the forward compression wave could be detected 1 minute after esmolol administration $(n=10)$ : peak intensity reduced by $30.3 \%(p=0.003)$ and was delayed $9.30 \mathrm{~ms}(p=<0.001)$.

Conclusion: This new method enables wave intensities, reflections and speeds to be obtained non-invasively at any ultrasound accessible site. It could provide a clinically useful way to detect heart failure, and alteration of arterial tone and stiffness.

\section{REFERENCES}

[1] Parker KH. Med Biol Eng Comput 2009;47:175-88.

[2] Curtis SL. Am J Physiol Heart Circ Physiol 2007;293:H557-H62.

[3] Feng J, Khir AW. J Biomech 2010;43:455-62.

(C) 2019 Association for Research into Arterial Structure and Physiology. Publishing services by Atlantis Press International B.V. This is an open access article distributed under the CC BY-NC 4.0 license (http://creativecommons.org/licenses/by-nc/4.0/). 